\title{
MDiET: Malware Detection in Encrypted Traffic
}

\author{
Dimitrios Schoinianakis \\ Nokia Bell Labs \\ Cyber Security Research \\ Werinherstr. 91, 81541 \\ Munich, Germany \\ dimitrios.schoinianakis@nokia-bell-labs.com \\ Norbert Goetze \\ Nokia Bell Labs \\ Data Science \\ Werinherstr. 91, 81541 \\ Munich, Germany \\ norbert.goetze@nokia-bell-labs.com \\ Gerald Lehmann \\ Nokia Bell Labs \\ Data Science \\ Werinherstr. 91, 81541 \\ Munich, Germany \\ gerald.lehmann@nokia-bell-labs.com
}

\begin{abstract}
With the increasing adoption of end-to-end encryption in industrial systems, the risk of distributing hidden malware by exploiting encrypted channels gradually turns to a major concern. Due to encryption, the stateof-the-art, signature-based mechanisms might fail to detect malware sufficiently, thus new approaches are required. In this work, a method for malware detection in encrypted traffic based on Machine Learning is presented. A supervised learning approach is adopted and the efficiency of the solution is demonstrated by a set of exhaustive simulations. Further considerations for incorporating the proposed method in a reference industrial network are also discussed.
\end{abstract}

malware, machine learning, supervised learning, loT, mobile networks, industrial automation

\section{INTRODUCTION}

In the era of $5 G$ and Industry 4.0 connectivity, the replacement of old, cable-based infrastructure with more versatile, wireless connections is gaining great momentum (H2020 ICT 2016 project 5G-MoNArch 2018). In the same context, the hardware as well as the underlying industrial protocols need to be revisited in order to facilitate the latest requirements for increased throughput, reliability, and security. After all, the convergence of IT and OT domains is now turning to a major concern not only for system architects but for security specialists as well (Pettey 2017).

Especially for security, things turn out to be more complicated. In the past, legacy industrial systems basically didn't have a security requirement since they were considered as closed systems and connectivity was mainly cable-based. This applied not only to the overall infrastructure but also to the associated protocols, e.g. PROFINET (PI North America).

Recent attacks towards critical, large-scale, industrial installations pinpoint exactly the core of the problem. The Stuxnet malware launched in 2009 against uranium enrichment centrifuges in Natanz, Iran, and its second wave against monitoring and control computer systems manufactured by Siemens (PLCs, Profibus modules, etc.) came as a shock to the security community (Zetter). The outcome of these attacks could have been detrimental, have the centrifuges been brought out of control. In 2017, one of the largest, global, and most coordinated cyber attacks with the use of "NotPetya" malware was launched, focusing initially on targets in Ukrainian territory and then spreading all around the globe. The result was more than $\$ 10$ billion in total damages (Greenberg). Lately, the "Triton" malware targeted industrial control systems manufactured by Schneider Electric (Newman). In specific, the malware cripples and manipulates the emergency shutdown protocols. If a malware can control such procedures there are many ways in which damages could be inflicted, for example environmental pollution by plants which are unable to control their waste management, power grid blackouts, or explosions (Newman).

Added to the complexity and sophistication of modern cyber-warfare, the expected adoption of end-to-end encryption creates yet another risk and 


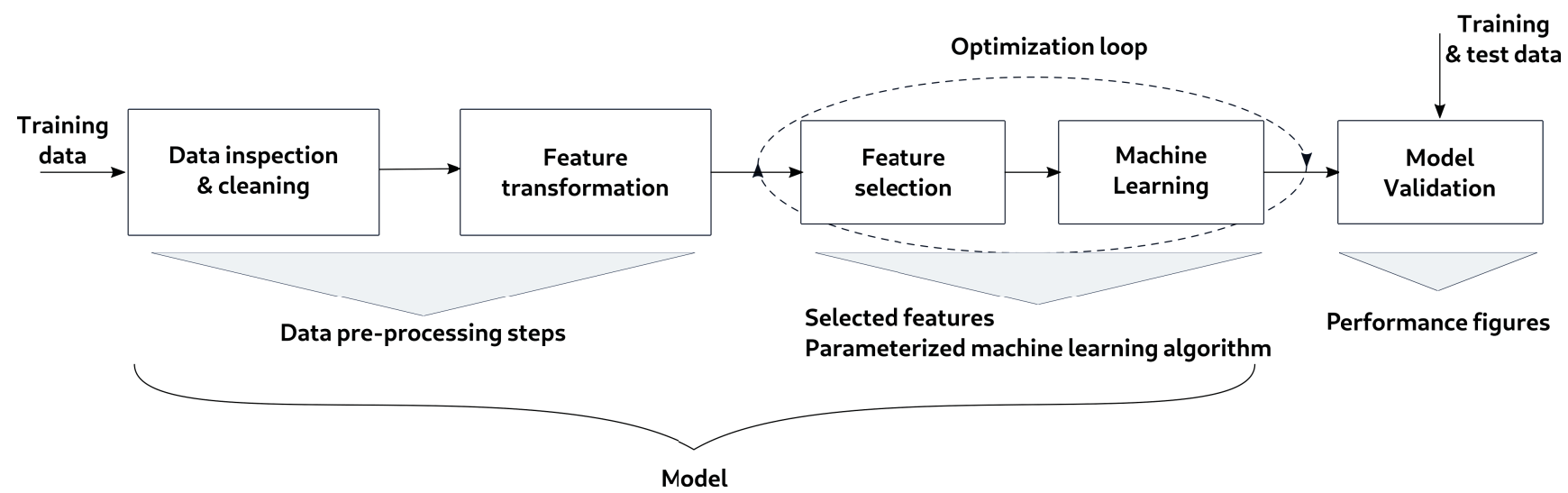

Figure 1: The general architecture of the Automated Model Builder

renders cyber-security an even more complex task. The point is that state-of-the-art, signature-based solutions might fail to detect newly delivered malware timely, since encryption by default plagues the detection capabilities of such systems. In this work, we attempt to mitigate the issue with the use of Machine Learning (ML) algorithms that are able to detect malware whose Command \& Control communications are encrypted.

The rest of the paper is organized as follows: in section 2 the test procedure and setup are presented, while in paragraph 2.1 a more detailed description of the core ML platform "Automated Model Builder" (AMB) is offered. The derived performance metrics are presented in section 3 and a suitable architecture to embed the proposed solution in a generic environment is discussed in section 4. Finally, conclusions are offered in section 5.

\section{METHODOLOGY}

\subsection{The Automated Model Builder}

At the epicentre of our method lies the Automated Model Builder (AMB), a proprietary tool developed in NOKIA Bell Labs. The AMB incorporates data science know-how and aims to build models without requiring the so-called domain knowledge as input. In this paper, domain knowledge means the knowledge of a security expert in order to label the metadata of the items under inspection. Therefore the AMB can be applied easily with the data as they were provided. A high-level architecture of AMB is offered in Figure 1 and the main processing steps include:

- Model: in this context, a model is a set of mathematical formulas defining the entire processing chain, e.g. pre-processing of data and the model calculation.
- Data Pre-Processing: in the data preprocessing stage, the data is prepared for machine learning.

* Data inspection \& cleansing: Filtering \& balancing of data samples: adaptation of the data set by removing or replacing samples and/or features (e.g. constant features or missing values).

Feature Reduction: reducing the number of features in order to reduce the processing time in the machine learning stage.

Feature Generation: this stage performs binning of numeric values and discretization of nominal values (e.g. E.g. discretization of numerical values or combination of features).

* Feature Transformation: this stage can normalize the features to include the information in which range these features reside in.

- Optimization loop: it searches for gain of combinations of selected features. The loop is using the performance values of the models to find optimal feature sets.

* Feature Selection

* Selection of machine learning algorithms

* Auto parametrization of machine learning algorithms

* Machine Learning

\section{- Performance Figures}

- Selection \& storage of the best models

- Model validation providing a wide range of performance figures

- Model explanations describing how malware is identified by a model 
The AMB generates, without human intervention, a set of best performing classification models which comprise not only the mathematical formula generated out of the machine learning stage, but also the formulas on how the data pre-processing was performed. Therefore, when applying a selected model, the AMB needs only to access the metadata which must have the same format as the training data with the exception, that features, unused by the model, can be omitted.

Besides the model itself, the AMB provides an evaluation result (precision and recall figures) of how well the detection will work, optimally itemized for specific malware types. In a further development, the results of the validation techniques of the AMB can be used to automatically decide, if a newly generated model should be deployed or not. For example, if a new model is built to cope with new malware types and, as a consequence, the recall values degrade, it can be decided to keep the previous model. This decision function may also include actual traffic and malware type statistics obtained from previous model applications to the current network traffic.

This is conducted to weight or even exclude specific malware types to achieve fair performance figures. If specific malware types represent a significant threat, but must be excluded from a new model for the sake of its performance, a second model specialized for the excluded malware type can be generated. This weighting technique is also applicable when a new training data set is generated to keep the detection performance focused and aligned to the current malware threat scenario.

\subsection{Test setup}

The general methodology to generate a suitable $\mathrm{ML}$ model is illustrated in Figure 2. Since a set of labeled data is required, we compiled a text file with 15.000 random https websites from a known list of the 1 million most popular domains (Amazon S3). A simple script loops through the text file and parses the websites as arguments to an instance of the headless version of Google Chrome browser. This generated a sufficient load of TLS traffic (approximately 10.000 TLS handshakes) and with the use of Wireshark tool (Wireshark) we obtained a set of benign traces in pcap format to serve as our benign labeled dataset. The complete setup was hosted in a Windows 7 virtual machine.

A similar set of approximately 242 pcap traces from real malware which establish SSL/TLS Command \& Control connections was collected from NOKIA's Threat Intelligence lab, to serve as the malign labeled dataset. The two datasets were then imported to Joy, an opensource tool developed by
Cisco, that extracts data features from network traffic (TCP header metadata, TLS metadata, etc) and exports them to JSON format (Cisco Systems). Joy may also be used as a packet sniffer and supports Netflow and IPFIX capture as well. Note also that TCP packets with a zero-length data field are not reported, hence not included in this work either.

The output from Joy is not compatible with the AMB, thus the JSON files generated by Joy need to be transformed to the appropriate format. This involves common data manipulation to generate a well-structured table that can be processed by AMB. The final table comprises basically rows each containing the information of TLS handshakes (most of which is not encrypted) along with IP and TCP data) and a notation of whether the respective row is a benign or malign sample. Moreover, because only a small portion of the IP packets are processed, an optimal resource utilization is achieved. Typical features included in the analysis are:

- number of packets (client and server)

- number of bytes (client and server)

- flow duration

- TCP flags

- packet distribution probability

- Sequence of packets' lengths and time

- TLS features (ordering of cipher-suites, version, advertised extensions, etc.)

Consequently, the AMB will perform the automated analysis and produce the models that achieve the highest possible performance for the respective dataset.

\section{PERFORMANCE}

The AMB performs autonomously an analysis of the labeled data and outputs several models along with a graphical representation that shows how stronly the auto-selected features contribute to the decision. In Figure 3 for example, a visual representation of the output of AMB is offered for two different models. Although the models refer to the same feature-set (i.e., all features tis_c_exts_server_name, tls_sni, tcp_out_first_window_size, packets_00_b, tcp_out_opts were excluded) the tool will generate several models using different feature combinations. Each colored bubble corresponds to a feature. The bigger the bubble is and the closer it is to the red bubble labeled as malign in the upper left corner, the greater is its contribution to the detection of malign samples. 


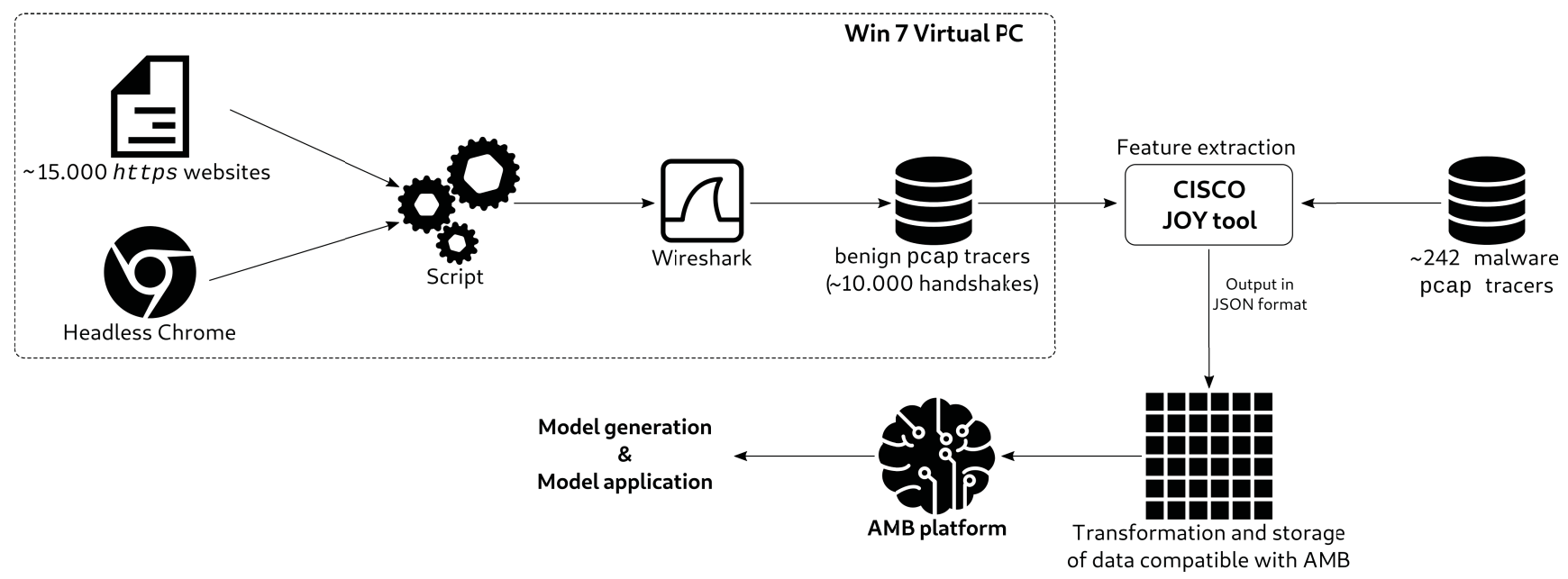

Figure 2: Test setup for model training/generation/application

Table 1: List of malware samples used for model application

\begin{tabular}{ccc}
\hline Platform & Type & Name \\
\hline Win32 & Downloader & Rovnix.H \\
Win32 & Downloader & Rovnix.H \\
Win32 & PasswordStealer & Ramnit \\
Win32 & PasswordStealer & Ramnit \\
Win32 & Trojan & ExtenBro.CN \\
Win32 & Trojan & ExtenBro.CN \\
Win32 & InfoStealer & DarkVNC \\
Win32 & InfoStealer & DarkVNC \\
Win32 & Keylogger & AgentTesla \\
Win32 & Adware & OKitSpace \\
Win32 & Adware & OKitSpace \\
Win32 & Spyware & NexusLogger \\
Win32 & Spyware & NexusLogger \\
Win32 & Downloader & JS.Agent.QCl \\
Win32 & Downloader & JS.Agent.QCl \\
Win32 & RansomWare & ShurLOckr \\
Win32 & RansomWare & ShurLOckr \\
Win32 & Miner & ComboJack \\
Win32 & Miner & ComboJack \\
Win32 & BankingTrojan & Metamorfo \\
\hline
\end{tabular}

What is worth mentioning here is that, although both models achieve very high detection rates, the model in Figure $3 a$ might not be the optimal one because it relies on many features and utilizes TLS-specific features, which are undesirable in this case (see 3.1). The model in Figure 3b, on the other hand, comprises only a few, non TLS-related features, hence it is an excellent candidate for model application.

After several experiments two suitable models were derived and they were applied to a fresh data-set containing a mix of malign and benign traffic to check their performance. The fresh samples are depicted in Table 1; note that family names are not well standardized within the AntiVirus industry, and the variant identifiers are even less so. As a result we typically choose the malware name that most closely aligns with the threat documentation/references.

The results of the model application are offered in Figures $4 \mathrm{a}$ and $4 \mathrm{~b}$ (in fact Figure $4 \mathrm{~b}$ corresponds to the model in Figure $3 b$ ). Both models achieve very high performance rates (Area Under Curve AUC) above $94 \%$, which dictates the efficiency of our approach. Note also the precision and recall graphs, where in our case precision (for the class of being malign) is the number of samples of correctly indicated malign samples divided by the number of samples being indicated as malign (i.e., Precision $=T P /(T P+F P))$, where $T P$ are the true positives and FP are the false positives. Recall (for the class of being malign) is the number of samples correctly indicated as malign divided by the number of all samples being malign (i.e., Recall = $T P /(T P+F N))$, where $F N$ are the false negatives.

As a final note, CISCO has also developed a commercialized platform to detect malware in encrypted traffic CISCO (2019); Anderson and McGrew (2016). The main difference with the proposed approach is that we do not require labour of skilled data scientists and the process adapts efficiently to changing malware in an autonomous manner while achieving the best possible malware detection accuracy. In general, the solution presented here supports an ongoing adaptation of the malware detection process in a very efficient way. This becomes feasible because:

1. Only the input of small, correctly labelled training data is needed, but no other parameters, settings or choices steering the model building process 
MDiET: Malware Detection in Encrypted Traffic Schoinianakis • Goetze • Lehmann

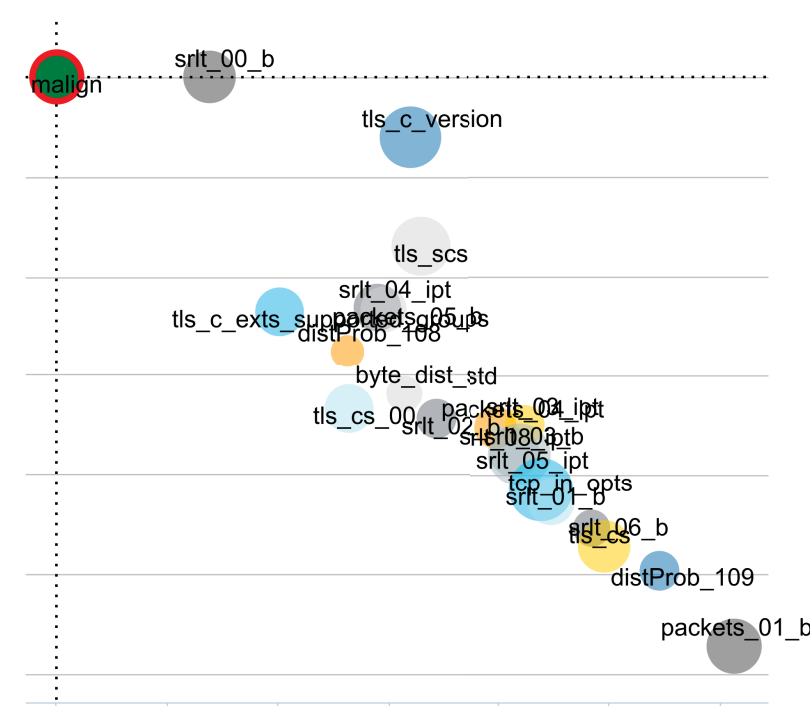

(a) Sub-optimal model with many features

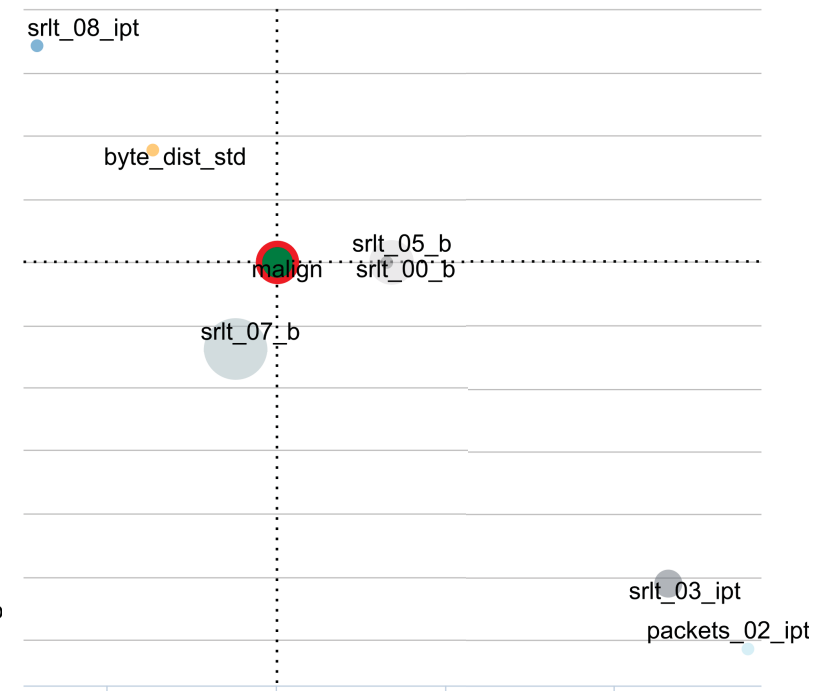

(b) Optimal model with a few, non-TLS features

Figure 3: Visual representation of the performance and feature sets of two models

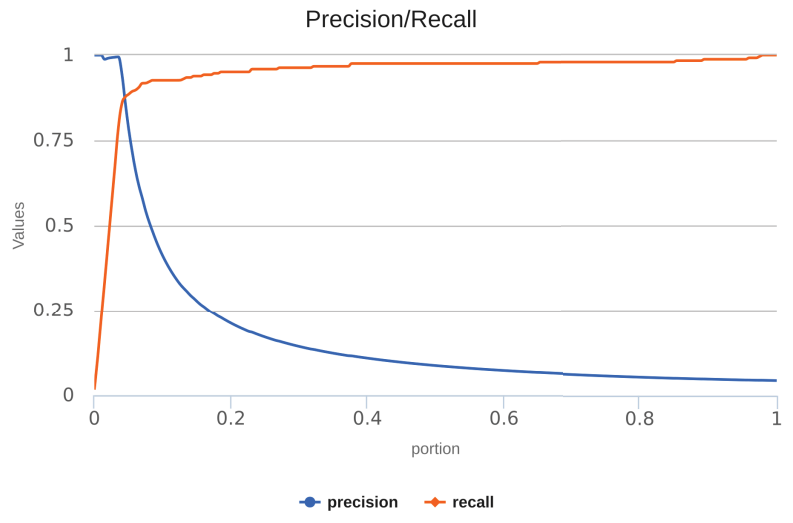

(a) AUC: 0.967 - Accuracy: 0.991

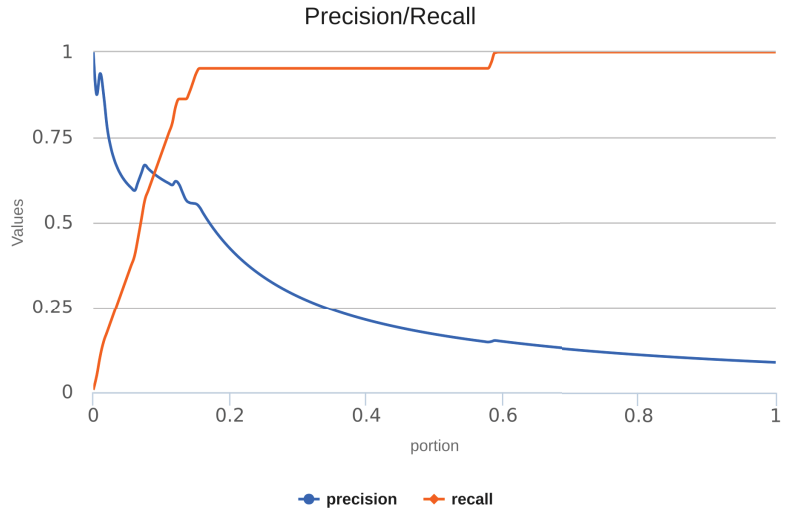

(b) AUC: 0.947 - Accuracy: 0.942

Figure 4: Two models applied to a fresh malware sample set

2. During application of the model, the performance is monitored considering the actual malware mix and re-training steps are triggered automatically

3. Very weak detections (decisions) are forwarded to operator and he needs to examine only those for improving/adapting the detector to new variants of malware

\subsection{Limitations}

Due to the fact that only one client machine was used to generate the benign traffic, all client-related TLS features were excluded from our analysis to minimize bias. The reason is that in our setup all benign traces were generated using the same machine and the AMB could falsely identify benign traffic by looking only for this machine's specific TLS fingerprint. Despite this limitation, the non-TLS features (basically TCP metadata) were sufficient to offer very high performance levels as described in the previous paragraph. Part of future work is to gain access to anonymized corporate data (i.e., multiple clients) to assess the effect of TLS-related features in the performance of the generated models.

\section{REFERENCE ARCHITECTURE}

In Figure 5, an end-to-end architecture for malware detection in industrial networks is illustrated. The AMB is an essential building block of the architecture allowing a quick adaptation to new malware without human intervention or manual data analysis work.

To allow for scalability, traffic data can be recorded at tapping points at the edges of the industrial network. For obtaining the metadata, the operator may employ 


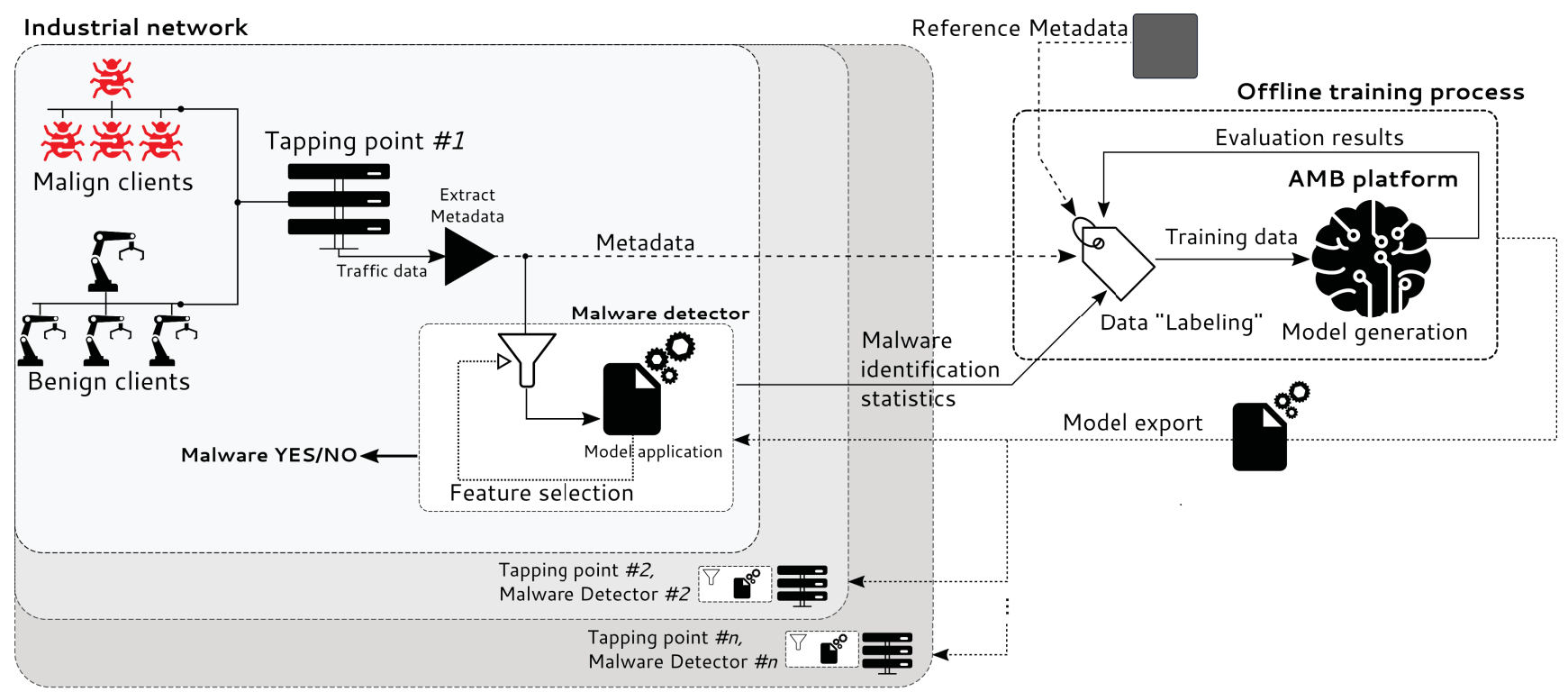

Figure 5: Reference architecture for industrial applications

standard packet capturing techniques, or utilize ondevice tools that are supported in a plethora of modern routers and switches. It is important to note that this metadata must be processed in the same way at all tapping points (to provide consistent data). The metadata serves as basis for both offline training and malware detection. Metadata has the advantage of being privacy-respecting, meaning that the application layer payload exchanged by the clients is not stored.

Still referring to Figure 5, the off-line training process encompasses known examples of malign traffic (true positives) and known examples of benign traffic (true negatives). The combination of both represents the 'Reference Metadata' which, when labeled accordingly, is referred to as Training Data. All available metadata of a data link should be offered during the training phase, i.e., there should be no manual pre-selection before offering the data to the AMB.

The use of a fully automated model building technique in the malware detector also exhibits several advantages. It allows the adaptation of the detector for new malware types in a faster and cheaper way, since human intervention by a skilled data scientist and/or network expert and/or IT security expert is not required. This is due to the approach to offer all metadata available for an encrypted data link to the model building process without performing any pre-selection. Hence, it is ensured that the AMB gets all information available to make the optimal feature selection. If there are specific patterns in the metadata common to a specific type of malware they will be automatically identified as a result of the training process.

In general, two model building approaches are possible: either build one model detecting all kinds of malware giving an indication if the data traffic originated from infected clients or not, and/or to build specific detector models focusing on specific malware types/families and then to deploy those models as multiple (specialized) detectors.

The output of the validation result of the AMB can also be used to inform the industry in more detail, which malware types can be detected optimally. Also, model explaining techniques incorporated in the AMB can be used to describe in a humanunderstandable way, how a detection model works in detail and what the decisive factors are.

Note that in Figure 5 the malware detector that inspects traffic data can forward or report a low number of traffic metadata samples, for which the detector determines a low confidence of the detection result ("malware identification statistics". For example, this could be the case for new malware derived from a former malware type. These forwarded examples could be reviewed by experts and, based on their decision, an additional tagged example set can be generated and added to the current training data set. This extended training dataset can be used to train a new model for removing non-confident detection results. By doing so, an incremental learning and accounting of new malware types can be achieved without the need to obtain knowledge of this malware type by external sources or by infection cases reported to the operator. With 
this, new malware types can be detected before a broader infection spread of client devices occurs.

\section{CONCLUSIONS}

A method and an architecture to detect malware in encrypted traffic with the use of Machine Learning have been presented in this work. The process to collect training data along with the intrinsic mechanics of the Automated Model Builder have been analysed and the detection rates under several selected models have demonstrated the efficiency of our method. Next steps include the adaptation of the proposed approach to an IoT and Industrial IoT environment, namely with simple smart devices and more complicated industrial robotics (PAiCE). The scope of future work is not only the detection of malware in an loT or IloT environment, but anomaly detection in general.

\section{REFERENCES}

Amazon S3. Top 1 million websites. URL http://s3.amazonaws.com/alexastatic/top-1m.csv.zip.

B. Anderson and D. McGrew. Identifying encrypted malware traffic with contextual flow data. In Proceedings of the 2016 ACM Workshop on Artificial Intelligence and Security, AISec '16, pages 35-46, 2016.

CISCO. Whitepaper: Encrypted traffic analysis, Jan 2019. URL https://www.cisco.com/ c/dam/en/us/solutions/collateral/ enterprise-networks/enterprisenetwork-security/nb-09-encrytd-trafanlytcs-wp-cte-en.pdf.
Cisco Systems. URL https://github.com/ cisco/joy.

A. Greenberg. The untold story of NotPetya, the most devastating cyberattack in history. URL https://www.wired.com/story/ notpetya-cyberattack-ukraine-russiacode-crashed-the-world/.

H2020 ICT 2016 project 5G-MoNArch. Deliverable D3.1: Initial resilience and security analysis, Jun 2018. URL https://5g-monarch.eu/smartsea-port-use-case/.

L. H. Newman. Menacing malware shows the dangers of industrial system sabotage. URL https://www.wired.com/story/tritonmalware-dangers-industrial-systemsabotage/.

PAiCE. Research projects. URL https: //www.din.de/en/innovation-andresearch/research-projects/industry4-0/paice-271784.

C. Pettey. When IT and operational technology converge, Jan 2017. URL https://www. gartner.com/smarterwithgartner/whenit-and-operational-technologyconverge/.

PI North America. PROFINET, industrial ethernet for advanced manufacturing. URL http://us . profinet.com/technology/profinet/.

Wireshark. URL https://www.wireshark. org/.

K. Zetter. An unprecedented look at Stuxnet, the world's first digital weapon. URL https: //www.wired.com/2014/11/count downto-zero-day-stuxnet/. 